\title{
Téoros
}

Revue de recherche en tourisme

\section{L’impact du tourisme sur les sites sacrés en Amérique précolombienne}

\section{L'exemple des sites rupestres amérindiens et inuits du Bouclier} canadien

\section{Daniel Arsenault}

Volume 16, numéro 2, été 1997

Tourisme et religion

URI : https://id.erudit.org/iderudit/1074575ar

DOI : https://doi.org/10.7202/1074575ar

Aller au sommaire du numéro

Éditeur(s)

Université du Québec à Montréal

ISSN

0712-8657 (imprimé)

1923-2705 (numérique)

Découvrir la revue

Citer cet article

Arsenault, D. (1997). L'impact du tourisme sur les sites sacrés en Amérique précolombienne : l'exemple des sites rupestres amérindiens et inuits du Bouclier canadien. Téoros, 16(2), 21-25. https://doi.org/10.7202/1074575ar d'utilisation que vous pouvez consulter en ligne. 


\title{
$+\ldots . .$. \\ L'IMPACT DU TOURISME \\ SUR LES SITES SACRÉS EN AmÉRIQUE PRÉCOLOMBIENNE
}

\author{
L'EXEMPLE DES SITES RUPESTRES AMÉRINDIENS \\ ET INUITS DU BOUCLIER CANADIEN
}

\begin{abstract}
Daniel Arsenault, chercheur en archéologie CELAT - Université Laval, Québec
\end{abstract}

Le tourisme culturel et scientifique, celui qui s'intéresse en particulier aux vestiges du passé, connat̂t un essor phénoménal dans le monde. Sur le continent américain, on trouve généralement, parmi les lieux les plus intéressants fréquentés par cette forme de fourisme, des sites à caractère sacré ou disposant de monuments à foncrions religieuses. Les plus achalandes des sites précolombiens sont, entre autres: Machu Picchu (inca) au Pérou, Tikal (maya) au Guatemala, Teotihuacan (culture du même nom) au Mexique, Mesa Verdez (anasazi) aux États-Unis. Chacun comporte une série de bâtiments à fonctions religieuses qui en font des sites sacrés. Comme il s'agit de sites de renommée internationale, les mesures de protection et les programmes de mise en valeur sont bien etablis et les budgets de fonctionnement annuels sont suffisants. Dans cet essai, je discuterai des sites sacrés autochtones que l'on trouve aux États-Unis et, surtout, au Canada et au Québec, afin de signaler les conséquences négatives que peuvent avoir les touristes. Les groupes autochtones s'inquiètent de plus en plus des menaces des touristes et des promoteurs sur les sites sacrés qu'ils fréquentent encore (Arsenault et Gagnon, 1996: 175; Mohs, 1996: 200). Je me concentrerai plus particulièrement sur des lieux sacrés de tradition algonquienne ou inuit situés dans le Bouclier canadien, formés par des sites de peintures ou de gravures rupestres (Arsenault et Gagnon, n.d.a) dont certains ont au moins un à deux millénaires (Arsenault et Watchman, n.d.). J'aborderai certaines conséquences de la fréquentation touristique et proposerai quelques solutions pour permettre d'harmoniser les nelations entre les groupes autochtones et les touristes. Je répondrai ainsi à quelques questions de la psychologue sociale Jane Hubert (1996: 11-12) sur la fréquentation des sites sacrés: Les touristes sont-ils conscients de l'effet qu'ils ont lorsqu'ils pénètrent dans des liewx aussi chargés symboliquement pour les communautes autochtones actuelles? Que font les responsables pour garantir que les sites fréquentés sont bien respectés par les visiteurs et que ces demiers comprenient les valeurs religieuses inhérentes? Il convient d'abord de voir ce qu'est un site sacré pour les groupes autochtones.

\section{QU'EST-CE QU'UN SITE SACRÉ ?}

Il est difficile de définir exactement un site sacré compte tenu de la diversité des cultures autochtones. Les sites sacrés n'ont généralement pas la visibilitế des sites historiques ou, du moins, ne livrent pas autant d'indices intelligibles pour le néophyte que des monuments à fonctions religieuses d'origine ou d'inspiration européenne. Quel que soit le groupe, on peut distinguer deux catégories de sites sacrés : aménagés et non aménagés. Dans la première catégorie, on trouve les cimetières et les structures architecturales à usage rituel : kiva des Anasazis du SudOuest américain (Brody, 1993), suerie des groupes algonquiens du Bouclier canadien (Paper, 1990). Dans la seconde, les sites ne présentent généralement aucune forme d'aménagement évident ; ils sont constitués par des éléments du paysage naturel, tels sommets de montagnes, cavernes, formations rocheuses, rivières ou bois. Dans certains, cependant, les seules manifestations symboliques produites par des agents humains sont les inscriptions rupestres (Arsenault, 1997 ; Carmichael, Hubert et Reeves, 1996: 1 ; Theodoratus et LaPena, 1996). On trouve done plus d'un type de sites sacrés, mais la frontière est parfois difficile à établir entre le lieu lui-même et le territoire environnant, qui peut également être sacré (Hubert, 1996: 16-18) ; c'est le cas, en particulier, des endroits où il y a des sites rupestres de facture algonquienne (Arsenault, 1997 ; Dewdney et Kidd, 1967 ; Rajnovich, 1994 ; Vastokas et Vastokas, 1973).

Quelle que soit la nature des sites sacrés (aménagés ou non), ils sont chargés de pouvoir (Arsenault, 1997 ; Arsenault et al., 1995 ; Carmichael, 1996 ; Mohs, 1996 : 197 ; Theodoratus et LaPena, 1996 : 22). Selon les croyances religieuses autochtones, ces lieux sont des points de jonction avec le monde non empirique, celui de l'esprit des ancêtres, celui des puissances ou des entités surnaturelles qui y résident (Conway et Conway, 1990 ; Gile, 1995). En outre, même si ces lieux peuvent être liés aux ancêtres d'un groupe donné - comme un cimetière -, plus que des foyers pour la pratique du culte des ancêtres, des lieux de commémoration (Carmichael, Hubert et Reeves, $1996 ; 6$ ), ce sont des endroits de ressourcement spirituel pour les pratiquants d'une religion autochtone (Price, 1996 : 261). De ce fait, la fréquentation 
des lieux sacrés exige des règles de conduite respectueuses (Arsenault et Gagnon, 1996 : 175 ; Carmichael, Hubert et Reeves, 1996:3) et le dépôt d'offrandes, le plus souvent du tabac (Paper, 1988). Tout individu qui pénètre en ces lieux devrait reconnaître leur caractère sacré et manifester la même attitude de deférence que s'ils pénétraient dans un temple. Pourtant, un tel comportement est loin d'être généralisế chez les touristes. On peut donc s'interroger sur les effets perturbateurs - même destructeurs - qu'a pu engendrer l'accroissement des visites sur les sites déjà connus an cours des dernières annés.

\section{LES EFFETS PERTURBATEURS DU TOURISME SUR LES SITES SACRÉS}

Les effets perturbateurs de la fréquentation d'un site sacré peuvent être de deux types : matériel et spirituel. Chacun est étroitement associé aux valeurs que peuvent véhiculer les sites sacrés. Pour mieux comprendre leur portée, on peut partir des quatre catégories de valeurs associées aux sites préhistoriques proposées par Stanley Price (1994: 285), qui sont aussi applicables aux sites sacrés autochtones : $1^{\text {s }}$ valeurs esthétiques et artistiques ; $2^{\circ}$ valeurs économiques et utilitaires : $3^{\circ}$ valeurs symboliques et séman-

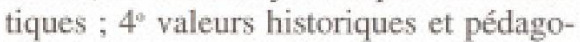
giques. L'exploitation touristique des sites sacrés met bien souvent en exergue certaines de ces catégories au détriment des autres, engendrant des problèmes matériels et spirituels.

\section{LES EFFETS PERTURBATEURS D'ORDRE MATÉRIEL}

Un des effets les plus pertubateurs observés est lié à la conservation. L'afflux croissant des touristes sur les sites peut gếnérer des problèmes aussi variés que discrets. La seule présence de visiteurs sur un site en plein air, sans aucune surveillance, peut provoquer des changements dans l'environnement immédiat, notamment lorsque ces visiteurs abandonnent sur place des déchets (mégot de cigarette, papier, canette). La fréquentation accrue d'un site sacré peut également entraîner des dommages accidentels aux infrastructures : usure des sentiers', ou bris des équipements d'accueil, occasionnant des frais de réparation ou de remplacement qui dépassent souvent les budgets disponibles. Ces éléments sont toutefois moins dommageables que le vandalisme et le pillage, actions nefastes que l'on peut observer même dans des endroits peu a médiatisés $\%$.

Au Québec, lé site de là Roche-âl'Oiseau, à la base d'une très haute falaise de granite sur la rivière des Outaouais, est sans contredit le plus imposant des sites québecois de peintures rupestres algonquiennes, le plus grand de tout le Bouclier canadien. Les parois omées qui couvrent le site s'étendent sur plus de 160 mètres de long et sur près de huit mètres de haut à la base d'une impressionnante falaise, haute de 150 mètres. Or, ce site constitue indubitablement l'un des exemples en art rupestre parmi les plus graves de vandalisme (Arsenault et Gagnon, n.da). La plupart des motifs préhistoriques peints à l'ocre rouge sur la surface rocheuse demeurent aujourd'hui recouverts par des graffitis - faits à l'aide de peintures en aérosol — que les plaisanciers (pour ne pas dire les plaisantins ! ! ) ont laissés au fil des ans (voir photo), ignorant vraisemblablement qu'ils étaient en présence d'un site rupestre préhistorique. Bien que connu. depuis des décennies par les habitants locaux ${ }^{4}$ - mais aussi par les touristes, si l'on en juge par l'indication du lieu d'origine de certains a taggeurs $w$ (ex. : * Texas s) - et répertorić depuis 1973 (Tassé, $1977: 38-44$ ), ce site n'a jamais bénéficié de programme de protection et ne dispose d'aucune infrastructure pour accueillir les visiteurs ou les avertir de l'existence d'un site sacré. Il faut cependant noter que les autochtones de la région semblent avoir délaissé ce site depuis des décennies, vraisemblablement en raison de sa désacralisation. Il aurait done fallu imposer des mesures de protection adéquates dès que l'existence du site fut rapportée aux autorités gouvernementales.

Ailleurs, le vandalisme peut se manifester sous la forme de vols d'offrandes laissées par les pratiquants autochtones (Reeves, $1996: 284-285$ ), par des marques peintes, rehaussées a la craie, au crayon gras ou au graphite, ou encore incisées pour faire ressortir certains motifs pour les photographier (Arsenault et Gagnon, n.d.a et b : Gagnon et Arsenault, 1996 ; Moly= neaux, $1985: 9$; Vastokas et Vastokas, $1973: 143$, note $14 ;$ Wainwright et Stone, 1992), voire même par le prélevement de portions ornées des sites comme

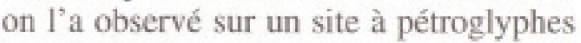
(gravures) du Nunavik (Arsenault et Gagnon, n.d.b ; Gendron, Arsenault et Gagnon, 1997 ; Saladin D'Anglure, 1962). Plusieurs interventions sont l'cuvre de touristes.

Un problème de taille demeure celui de la mise en valeur massive de certains sites sacrés qui privilégie bien souvent le profit economique et la surexploitation touristique. Il est reconnu que le tourisme culturel et scientifique représente une source sans cesse croissante de revenus pour divers paliers de gouvernements et deviennent même parfois très bénéfiques pour l'économie d'une région. L'un des exemples aujourd'hui les plus controversés est celui du site à pétroglyphes (appelé Teaching Rock) de Peterborough où, pour permettre aux visiteurs de contempler le site à l'abri et pour préserver les vestiges, on a érigé, en 1984, an cout de $800000 \$ \mathrm{CAN}$, une enorme structure couverte protégeant les quelque 900 motifs gravés sur des affleurements rocheux, sans suffisamment tenir compte des modifications de température que la fréquentation touristique dans une aire fermée allait engendrer ${ }^{t}$. II semble que ce soit ici davantage pour son potentiel touristique - le site reçoit parfois jusqu'à 200 visiteurs (Bahn, Bednarik et Steinbring, 1995 : 36) — plutôt que pour la valeur scientifique que recelait ce site, que des organismes subventionnaires ont privilégié une mise en valeur par trop ostentatoire. Cette démarche mettait de l'avant la valeur cconomique sous prétexte de donner préséance aux valeurs esthétique et pédagogique.

Exploiter un site sacré d'abord pour des raisons économiques et touristiques risque de mettre en valeur exclusivement les vestiges les plus attrayants, rendant problématique la connaissance archéologique plus approfondie du contexte historique. Ainsi, bien que le public obtiendra un aperçu spectaculaire du site, il sera moins bien compris puisque l'on aura négligé certains aspects dans le processus de mise au jour des vestiges (Stanley Price, 1994 : 286). Parfois, si l'interprétation des vestiges sacrés n'est pas suivie selon les normes professionnelles, les. 


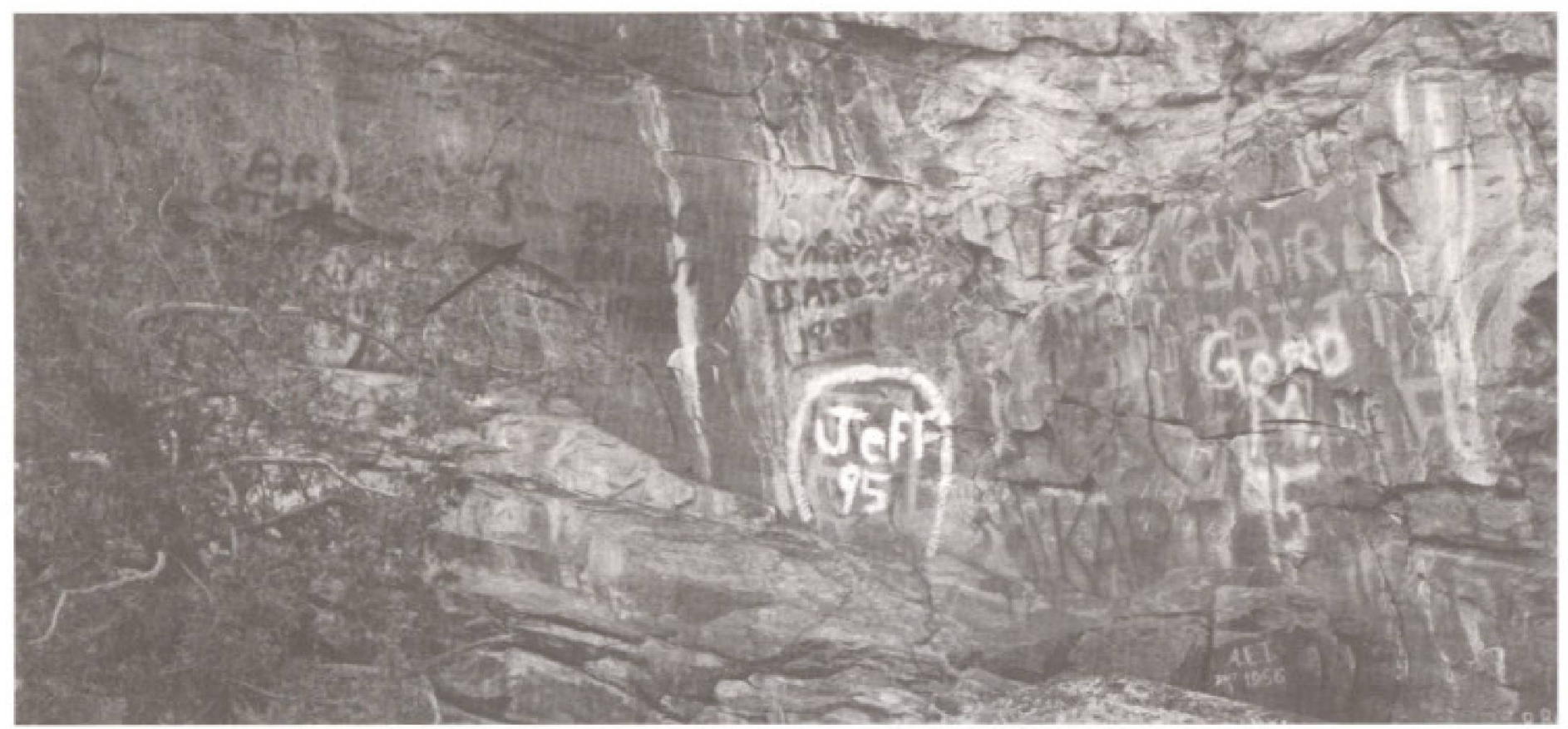

Exemple de vandalisme par graffiti sur le site de la Roche-d̀-l'Oiseau, sur la rivière des Outaouaïs. La plupart des motïfs tracés à l'ocre rouge et vraisemblablement de facture algonquienne et d'äge prehistorique ont été recouverts, au cours des dernières décennies, par les graffitïs laissés par les visiteurs. À noter cependant (flèche noire) les deux motifs superposés de poisson qui sont visibles à la gauche des prénoms Brad et Brent dans la portion cenire ganche de l'image.

touristes risquent de se faire dépeindre à la place un tableau spéculatif des activités religieuses, voire de se contenter de lectures strictement fantaisistes des contextes passés, non appuyées par des résultats scientifiquement contrôlés (Stanley Price, 1994 : 286). L'un des exemples les plus probants s'observe présentement en Estrie, avec l'exploitation risible, mais rentable, de sites à pétroglyphes et de $\alpha$ cairns $\%$, plus vraisemblablement de facture historique que préhistorique, que des amateurs font visiter aux touristes, prétendant y faire voir un site d'origine celtique (site de Potton, a Vale Perkins), et un site viking ? II est dommage de constater que des journalistes encouragent cette situation farfelue en rédigeant des articles dépourvus d'un minimum de sens critique et sans contre-vérification auprès de spécialistes (pour des exemples de couverture journalistique sur cette pratique d'archéologie fantasque, voir Baillargeon, 1995 ; Normand, 1996 ; SaintGermain, 1993).

\section{LES EFFETS PERTURBATEURS D'ORDRE SYMBOLIQUE}

Avec l'évocation de certains effets perturbateurs matériels, il devient plus aisé maintenant de saisir la portée des effets perturbateurs symboliques engendrés par des touristes sur les sites sacrés autochtones. On a vu la crainte des groupes spiritualistes amérindiens que les agissements de certains touristes (vols des offrandes laissées par des fidèles, vandalisme) conduisent ầ la désacralisation des sites habités par les esprits (Hubert, 1996 : 13-16 ; Reeves, $1996: 289$ ). Ces sites perdent alors leur fonction essentielle de permettre les échanges entre le monde matériel et le monde spirituel, car ils deviennent insignifiants. C'est probablement l'une des raisons qui explique que les communautés amérindiennes qui vivent dans la région du site de la Rocheà-l'Oiseau l'aient délaissé après avoir constaté que les parois ornées avaient été profanées.

L'ouverture au tourisme, et la * publicisation $s$, des sites sacrés autochtones peut entraîner une autre forme de profanation, motivée par des sentiments sectaires liés à certaines idéologies religieuses, en fournissant des lieux propices aux manifestations de groupes prêchant le Nouvel Âge (Ucko, 1994 : xvii). Il arrive mème parfois, qu'emportés par un prosélytisme icônoclaste, certains individus s'introduisent dans les sites sacrés, qu'ils considèrent comme * paiens $\%$, pour en altérer le contenu en laissant des messages explicites destinés à combattre les représentations religieuses non conformes à l'enseignement chrétien qu'ils prônent. Ce genre de pratique s'observe sur le site à pétroglyphes de Qajartalik, dans la baie d'Hudson, où des prosélytes ont marqué leur passage en incisant, sur certaines parois ornées, le signe de la croix et en inscrivant des mots sur Jésus (Arsenault et Gagnon, n.d.b).

On peut penser également que l'ordre du jour imposé par la période d'affluence touristique peut entrer en conflit avec celui des groupes autochtones désireux de pratiquer les rites ancestraux sur ces sites. Pour certains groupes les moments les plus propices pour conduire un rituel sur un site sacré peuvent correspondre à la haute saison touristique (Price, 1996). Il est important de trouver des solutions qui puissent satisfaire l'ensemble des groupes concernés.

\section{DES SOLUTIONS À ENVISAGER}

Bien que l'on ne puisse nier que certains sites sacrés appartiennent au patrimoine mondial et doivent être accessibles au public, cette condition ne doit pas aller à l'encontre de la stabilité de leur conserva- 
tion et du respect qu'ils méritent. Considérons quelques solutions qui pourraient garantir une meilleure préservation des sites connus tout en favorisant une attitude respectueuse de la part des touristes.

Il est évident que les organismes concernés doivent prendre conscience de la nécessité de développer des programmes d'éducation pour mieux informer le public sur la nature des sites sacrés autochtones. Ces programmes doivent comprendre des prescriptions - comment approcher les sites et attitudes à adopter sur place -, mais aussi des proscriptions - interdire l'accès à certaines zones ou à certaines périodes réservées aux rites autochtones. Il serait également utile de mettre en évidence, par le biais de panonceaux explicatifs par exemple, les conséquences que le tourisme a pu avoir sur le site fréquenté ou sur des sites similaires, pour sensibiliser les visiteurs à la conservation et aux problèmes de profanation. Comme l'écrit Stanley Price (1994), « les visiteurs devraient recevoir l'information sur l'état actuel du site et sur son évolution, tant d'un point de vue archécologique que sur le plan du paysage (naturel et culturel) dans lequel il a été inscrit. Plus le public sera conscient du processus de fabrication d'un site pour le faire connaître, plus il y participera et plus celui-ci lui paraîtra intelligible et sera susceptible d'être mieux préservé \% (traduction libre, ibid. : 288). Il faut souhaiter qu'un plan général de protection des sites sacrés soit développé et que des lois sévères soient promulguées par les tous gouvernements concernés, notamment au Québec, afin de garantir le développement harmonieux des programmes proposés.

La mise sur pied de tels programmes doit se faire dans la concertation totale, en privilégiant la participation de représentants des communautés autochtones dans les discussions sur l'interprétation des sites sacrés et sur les programmes de conservation et de mise en valeur (Creamer, 1994 ; Lowenthal, 1994 : 310-311). Cela suppose en outre l'incorporation, dans les politiques sur le patrimoine autochtone, des valeurs et des préoccupations des Amérindiens à l'égard des sites créés par leurs ancêtres. Une telle ouverture des promoteurs touristiques ne sera sans doute pas facile à obtenir, mais ces acteurs sociaux doivent établir des relations harmonieuses avec ceux dont ils veulent promouvoir les vestiges ancestraux. On peut espérer que ces programmes encourageront les changements d'attitudes des touristes à l'égard des cultures autochtones.

Pour des besoins de conservation et de respect des traditions, il faut également garder à l'esprit la possibilité de restreindre l'accessibilité aux sites en diminuant le nombre de visiteurs et en balisant les sentiers (Reeves, $1996: 288-289$ ). II faudra accepter l'idée que des communautés autochtones pourront parfois interdire l'accès public à certains sites (Mohs, 1996 : 202 ; Price, 1996 : 262). Les touristes, désormais mieux informés, seront en mesure de bien comprendre la portée de telles actions.

Malgré toutes ces solutions, il est utopique de penser que tout site sacré pourra être préservé du vandalisme ou d'un usage inappropriế (Carmichael, 1996 : 96), mais il faut espérer que des programmes d'éducation et de conservation seront efficaces en réduisant au maximum la détérioration ou la profanation des sites autochtones.

Un comité de lecture a lu et accepté ce texte

$\frac{-12}{15}$

\section{NOTES}

1 Jutilise le terme * précolombien * pour signifier la longue période historique précédant la venue de Christophe Colomb sur le continent américain, en 1492, qui allait marquer le début de l'implantation de colonies européennes au Nouveau Monde. Mẻme si parfois l'expression a Amérique précolombienne * renvoie strictement aux sociétés andines (Incas, Nazcas, Moche, etc.) et mésoaméricaines (Aztèques, Mayas, Olmeques, etc.), je réfère à l'ensemble des sociêtés autochtones qui ont peuplé l'Amé= rique entière depuis les origines, il $y$ a 12000 a 15000 ans.

2 Pour donner une idée de l'ampleur de la fréquentation touristique dans de tels sites, celui de Mesa Verde, au Colorado, recevait pas moins de 742000 visiteurs en 1992 et 666000 l'année suivante (Bahn, 1996 ; 370). Je n'ai pas les tableaux statistiques qui permettraient d'évaluer cette fréquentation pour les sites sacrés les plus connus du continent américain.

3 L'usure peut également être observée sur les surfaces ornées de certains sites rupestres, surtout si ces surfaces sont horizontales et peuvent âtre piétinées. C'est un phénomène que j'ai pu constater lors d'une visite exploratoire du site à pétroglyphes (gravures) du cap Qajartalik, sur l'île de Qikertaaluk, dans le détroit d'Hudson, en compagnie de collègues de l'Institut culturel Avataq, un organisme inuit. Ce site a reçu la visite périodique de touristes qui venaient fouler les affleurements de stéatite ornés de visages et de masques humains, sous la conduite de guides-archéologues pas toujours conscientisés aux problèmes de conservation inhérents à ce type de site archéologique (Gendron, Arsenault et Gagnon, 1996).

4 Ce site sacré fut mentionné pour la première fois par le Chevalier de Troyes (1918 [1686] : 36-37). Cet explorateur relate que les Amérindiens qui passent près de ce rocher ont coutume $\mathrm{d}^{+} \mathrm{y}$ lancer des flèches au bout desquelles ils attachent du tabac. Troyes ne semble toutefois pas avoir remarqué la présence de peintures rupestres à cet endroit.

5 Joan et Roman Vastokas ( $1973 ; 143$, note 14) rapportent que les principaux motifs gravés visibles sur le site à pétroglyphes de Peterborough, en Ontario, ont été rehaussés an crayon pour permettre aux visiteurs de mieux les apprécier....

6 Pour une critique du concept architectural proposé pour préserver le site de Peterborough, voir Bahn, Bednarik et Steinbring, 1995 ; les commentaires et répliques donnés par Bullen, 1996 ; Franklin, 1996 ; Wainwright, 1996 et la réponse finale de Bahn, Bednarik et Steinbring, 1996).

7 J'ai fait une critique détaillée de ce genre d'interprétation des sites à pétroglyphes (Arsenault, 1993).

\section{BIBLIOGRAPHIE}

Arsenault, Daniel (1993), Les pétroglyphes du site Jones de Vale Perkins, Canton de Potton. Evaluation critique des diverses theses proposées et discussion a propos des significations à donner aux gravures rupestres de ce site. Rapport inédit déposé au ministère de la Culture et des Communications du Quebec. Direction de l'Estrie, Sherbrooke.

Arsenault, Daniel (1997), \& Vers une archéologie du paysage au Québec. Esquisse du paysage sacré algonquien par le biais d'une étude contextuelle des sites rupestres préhistoriques du Bouclier canadien $\%$, à paraitre dans Recherches amérindiennes au Québec, XXVI, 3.

Arsenault, Daniel, et Louis Gagnon (1996), \& Le site Nisula (DeEh-1), Haute-Côte-Nord du Québec. Le défi de la conservation s, dans C. Mousseau (dir.), La Conservation In Situ / Archaeological Remains. In Situ Preservation, Montréal, Actes du 2e Congrés international ICOMOS (ICAHM Montréal 1994), p. 67-177. 
Arsenault, Daniel, et Louis Gagnon (n.d.a), * Splendeurs et misères des sites rupestres québécois. Bilan des recherches en cours et considération de l'état de conservation des sites à pictogrammes et à pétroglyphes au Québec $w_{\text {. }}$ Manuscrit en possession des auteurs.

Arsenault, Daniel, et Louis Gagnon (n.d.b.), w Le site a pétroglyphes du Cap Qajartalik. Résultats de la visite exploratoire de l'été $1996 \%$, Texte soumis à Etudes / Inuit / Studies.

Arsenault, Daniel, et Alan Watchman (n.d.) * Dating and Analyzing a Pictograph-Site in the Far-Eastern Canadian Shield : the Nisula site (DeEh-1) \%. Manuscrit en possession des auteurs.

Arsenault, D., L. Gagnon, C. A. Martijn, et A. Watchman (1995), o Le projet Nisula : recherche pluridisciplinaire autour d"un site à pictogrammes (DeEh-1) en Haute-Côte-Nord $x_{\text {. }}$ dans A.-M. Balac, C. Chapdelaine, N. Clermont et P. Desjardins (dir.), Archeologies quebecoises, Montréal, Recherches Amérindiennes au Québec (série ơ Paléo-Québec m, no 23), p. 7-57.

Bahn, Paul G., Robert G. Bednarik, et Jack Steinbring (1995), * The Peterborough petroglyph site : reflections on massive intervention in rock art i, Rock Art Research 1995, 12, 1, p. 9-41.

Bahn, Paul G., Robert G. Bednarik, et Jack Steinbring (1996), \& Peer review of massive intervention in rock art management practice $*$, Rock Ant Reseanch 1996. 13, 1, p. 54-60.

Bahn, Paul G., dir. (1996), The Cambridge Mustrated History of Archaeology, Cambridge, Cambridge University Press.

Baillargeon, Stéphane (1995), * Mystère irlandais à Mansonville P.Q. Une thérie fondée sur la découverte d'inscriptions celtiques veut que les ancêtres des Irlandais aient visité l'Estrie il y a 2000 ans $*$, dans l'édition du 20 mars du journal Le Devoir, cahier A, p. 1 et 8.

Brody, Jerry R. (1992), Les Anasazis. Les premiers Indiens du Sud-Ouest americain. Paris, Edisud.

Bullen, Margaret (1996), « Who's right? Whose right ? ${ }^{*}$, Rock Art Research, 13, 1, p, 47-48.

Carmichael, David $\mathrm{L}_{1+}$ Jane Hubert, et Brian Reeves (1996), Introduction $\%$, dans D.L. Carmichael, J. Hubert, B. Reeves et A. Schanche (dir.), Sacred Sites, Sacred Places, Londres et New York, Routledge (collection One World Archaeology, 23), p. 1-8.

Chevalier de Troyes (1918 [1686]), Journal de lexpedition du Chevalier de Troves à la Bale d'Hudson, en 1686 (Édition annotée par l'abbe Ivanhoê Caron), Beauceville, L'Éclaireur.

Conway, Thor, et Lulie Conway (1990), Spirits on Stone. The Agawa Pictographs, San Luis Obispo, Heritage Discoveries.

Creamer, Howard (1994), \&s Aboriginal perceptions of the past : the implications for cultural resource management in Australia w, dans $\mathrm{P}$. Gathercole et D. Lowenthal (dir), The Politics of the Past, Londres et New York, Routledge (collection One World Archaeology, 12), p. 130-140.
Dewdney, Selwyn, et Kenneth E. Kidd (1967), Indian Rock Paintings of the Great Lakes, 2 bd. Toronto, University of Toronto Press (Quetico Foundation).

Franklin, Nathalie (1996), * An Australian perspective of the Peterborough project $s_{1}$ Rock Art Research, 13, 1, p. 48-49.

Gagnon, Louis, et Daniel Arsenault (1996), Evivaluation des portions gravées prélevées sur le site à pétroglyphes de Bromptonville et conservées au Musée du Séminaire de Sherbrooke. Manuscrit inédit déposé à la Direction de l'Estrie, ministère de la Culture et des Communications du Quebec, Sherbrooke.

Gendron, Daniel, Daniel Arsenault. et Louis Gagnon (1996), \& A propos du projet de sauvetage des pétroglyphes de Qajartalik. Réplique à la lettre de M. Patrick Plumet $*$, Etudes / Inuir / Studies, 20, 2, p. 117-122.

Gile, Marie A. (1995), The Thunderbird and Undermater Panther in the Material Culture of the Great Lakes Indians : Symbols of Power. Mémoire de maîtrise déposé à la Michigan State University, East Lansing.

Hubert, Jane (1996), * Sacred beliefs and beliefs of sacredness $\$$, dans D.L. Carmichael, J. Hubert, B. Reeves et A. Schanche (dir.), Sacred Siles, Sacred Places, Londres et New York, Routledge (collection One World Archaeology, 23). p. 9.19.

Hygen, Anne-Sophie (1996), « Conservation, intervention or destruction of rock art ? Some Scandinavian experiences $\%$, Rock Art Research, 13,1, p. $48-52$.

Lowenthal, David (1994), "Conclusion : archaeologists and others $x_{\text {, dans }}$ P. Gathercole et D. Lowenthal (dir.), The Politics of the Past, Londres et New York, Routledge (collection One World Archaeology, 12), p. 302-314.

Mohs, Gordon (1996), * Sto : $l^{\circ}$ sacred grounds *, dans D.L. Carmichael, J. Hubert, B. Reeves et A. Schanche (dir.), Sacred Sites, Sacred Places, Londres et New York, Routledge (collection One World Archaeology, 23), p. 184 208.

Molyneaux, Brian (1985), \& Floating the Micmac vision s, Rotunda, été 1985, p. 6-11.

Normand, François (1996), * Une inscription qui suscite l'intérêt. Des caractêres runiques gravếs dans la pierre ont été découverts en 1995 \%, dans l'Édition du 15 août du journal Le Devoir, cahier B, p. 1.

Paper, Jordan (1988), Offering Smoke : The Sacred Pipe and Native American Religion, Moscow, University of Idaho Press.

Paper, Jordan (1990), \& 'Sweat-Lodge' : A Northern Native American Ritual for Communal Shamanic Transe *, Temenos, 26, p. 85-94.

Price, Nicole (1996), \& Tourism and the Bighorn Medicine Wheel : how multiple use does not work for sacred land sites $"$, dans D.L. Carmichael, J. Hubert, B. Reeves et A. Schanche (dir.), Sacred Sites, Sacred Places, Londres et
New York, Routledge (collection One World Archaeology no 23), p. 259-264.

Rajnovich, Grace (1994), Reading Rock Art. Interpreting the Indian Rock Paintings of the Canadian Shield. Toronto, Natural Heritagel Natural History Inc.

Reeves, Brian (1996), * Ninaistakis - the Nitsitapii's sacred mountain : traditional Native religious activities and land use/tourism conflicts ", dans D.L. Carmichael, J. Hubert, B. Reeves et A. Schanche (dir.), Sacred Sites, Sacred Places, Londres et New York, Routledge (collection One World Archaeology, 23), p. 265295.

Saint-Germain, Michel (1993), * Obélix en Amérix. Des pierres récemment découvertes remettent en cause I'histoire officielle. Mégalithes celtiques ou artefacts amérindiens ? , dans L'actualité, numéro du 1* mai 1993, p. 41-45.

Saladin D'Anglure, Bernard (1962), * Découverte de pétroglyphes à Qujartalik sur l'île de Qikertaluk *, North/Nord, numero de novembredécembre, p. 34-39.

Stanley Price, et Nicholas P. (1996), * Conservation and information in the display of prehistoric sites , dans P. Gathercole et D. Lowenthal (dir.), The Politics of the Past, Londres et New York, Routledge (collection One World Archaeology, 12), p. 1-8.

Tassé, Gilles (1977), \& Premières reconnaissances $*$, in G. Tassé et S. Dewdney, eds, Relevés et travaus récents sur l'art rupestre amérindien, Montrúl, Laboraloire d'archéologie de l'Université đu Québee à Montréal (Collection PaléoQuébec 8), p. 35-69.

Theodoratus, Dorothea J., et Frank LaPena (1996). * Wintu sacred geography of Northern California $\%$, dans D.L. Carmichael, J. Hubert, B. Reeves et A. Schanche (dir.), Sacred Sires, Sacred Places, Londres et New York, Routledge (collection One World Archaeology, 23), p. 2031 .

Ucko, Peter J. (1994), * Foreword \%, dans P. Gathercole et D. Lowenthal (dir), The Politics of the Past, Londres et New York, Routledge (collection One World Archaeology no 12), p, ix-xxi.

Vastokas, Joan, et Romas Vastokas (1973), Sacred Art of the Algonquins : A Study of the Peterborough Petroglyphs. Peterborough, Mansard Press.

Wainwright, Ian N.M. (1996), * Structure protects rock art in Petroglyphs Provincial Park, Ontario, Canada *, Rock Art Research, 13, 1 , p. $52-53$.

Wainwright, Ian N.M., et Thomas G. Stone (1992) * Ce fléau des graffiti sur les cuvres d'art rupestre $n$, Bulletin de l'Institul canadien de Conservation, 9, p. 4-6. 